\title{
No difference in the failure rates of orthodontic brackets bonded with self-etching primer
}

\author{
Are there more orthodontic bracket bond failures with self-etching primer \\ than with conventional etch and primer systems?
}

\section{Banks $\mathbf{P}$, Thiruvenkatachari $B$. \\ Long-term clinical evaluation of bracket failure with a self-etching primer: a randomized controlled trial. J Orthod 2007; 34:243-251}

Design This was a randomised controlled clinical trial (RCT) in a UK district general hospital.

Intervention The interventions tested were orthodontic brackets bonded with standard light-cured hydrophobic primer [acid etch (AE); Transbond XT, 3M Unitek, Monrovia, California, USA] compared with self-etching primer (SEP; Transbond-Plus, 3M Unitek). Both groups used Transbond XT paste(3M Unitek) and curing light.

Outcome measure The primary outcome was first-time bracket failure recorded by date and tooth number. Secondary outcomes were mandibular and maxillary bracket failures and the time taken to bond the bracket.

Results There were no statistical differences in failure rates between SEP (4.8\%) and AE (3.5\%). Bonding time per bracket with SEP was significantly lower at $75.5 \mathrm{~s}$ (95\% confidence interval, 72.9-78.5) in the SEP compared with the AE group (97.7 s; $95 \%$ confidence interval, 94.3-101.2).

Conclusions There was no difference in the failure rates of brackets bonded with either SEP or conventional AE using Transbond XT paste. Bonding with SEP was significantly faster than using conventional AE.
Address for correspondence: Phil Banks, Orthodontic Department, Fairfield General Hospital, Bury, Greater Manchester BL9 7TD, UK. E-mail: philbanks_burnly@yahoo.com.

\section{Commentary}

Knowledge is the common thread in all human endeavours, driving change and following the testing of even established truths. It is an integral part of science to re-evaluate, look for flaws and even selectively police existing strategies and methods. This study on the clinical evaluation of SEP in bracket failure is an endorsement of that process.

The authors have borne in mind the existing evidence, which includes a systematic review on bracket adhesives by Millett and others ${ }^{1}$ and a backdrop of divergent studies on SEP and reasons for bracket failure, when setting up their RCT. The objectives and outcomes of the trial are focussed and clear. The null hypothesis proposed is simple and direct with the primary aim of the study being a comparison of bracket failure after use of SEP and AE over a period of active treatment. Secondary derivatives of the study are the factors that contribute to bracket failure and efficiency of SEP in terms of time.

The elegant simplicity and well-defined outcomes makes the structure of the study precise, with clear inclusion and exclusion criteria, sample size selection and randomisation. The clinical methods have been standardised, including the use of the same light cure with a periodic check on the output, a point that gets missed in day-to-day practice. The weakness of the study was in fact delineated by the authors: it relates to the gap in the study design wherein the clustering of failed brackets in the same patient is not taken into account, although the sample size and statistical methods (Kaplan Meier and linear regression analysis) would avoid this and strengthen the results.

The outcomes here are clear: both bonding systems are satisfactory and failure and survival rates are similar, confirming the null hypothesis. They also conform to the previously published results in literature. The fact that bracket failure rate was not influenced by the bonding method, age, sex, left or right quadrants, nor upper or lower jaw, seems to indicate that the crux of bonding success is the material and a standardised technique. A clear biological perspective emerges: shallower resin tag penetration and less enamel demineralisation corresponding with failure when using SEP indicates that the key feature is the enamel-adhesive interface. If the bonding failures are the same with less enamel demineralisation, then the SEP may be a better alternative for efficient bonding than the AE technique.

A 7-min timesaving over the bonding of 20 teeth, indicated by this evidence, is also clinically relevant. The need for a pumice prophylaxis does not constitute an important difference between the two techniques as the $\mathrm{AE}$ group of patients may in any case present with teeth that are stained and not visually clean.

Historically, it has been presumed that the primary mechanism for retention during bonding is the mechanical interlocking between the adhesive and the enamel. It was also presumed that aggressive etching, such as that using 32\% orthophosphoric acid, might produce better bond strength. It has been observed 
subsequently by various researchers that shallow, less defined etching patterns produced by SEP provide just as adequate bond strengths. Low-pH SEP with minimal etching may provide adequate bond strengths, comparable to AE bonding, which strongly supports the use of SEP in orthodontic practice.

The authors have arrived at the conclusion that, although bracket placement time is significantly reduced, there is no difference in the failure rates or survival time of brackets bonded with Transbond XT paste using either Transbond Plus SEP or conventional etch and XT primer. The study is welcome as it reaffirms the need to continuously update knowledge on existing materials and methods, clarifying our perceptions of the issues. The authors are to be complimented on the simple yet focussed research which provides us with valid scientific evidence. The search for truth is never complete: continuous scepticism and re-evaluation of our experiences is the basis for scientific advancement.

\section{Practice point}

SEP are an efficient and effective alternative to the AE technique in orthodontic bonding.

\section{Anmol Khalha}

Department of Orthodontics, Institute Of Dental Studies and Technology, Delhi, India.

1. Millett DT, Glenny AM, Mattick CR, Hickman J, Mandall NA. Adhesives for fixed orthodontic bands. Cochrane Database Syst Rev 2007; issue 2.

Evidence-Based Dentistry (2008) 9, 79-80. doi:10.1038/sj.ebd.6400598 